\title{
Age-associated features of oxidative stress as marker of vascular aging in comorbid course of hypertension and type 2 diabetes mellitus
}

\author{
Valeriya Nemtsova1, Olexander Bilovol' ${ }^{1}$, Irina Ilchenko, Anna Shalimova² \\ 1The Clinical Pharmacology and Internal Diseases Department, Kharkiv National Medical University, Kharkiv 61039, Ukraine. \\ ${ }^{2}$ Department of Chronic Non-communicable Disease Prevention, Government Institution "National Institute of Therapy named \\ by L.T. Malaya of National Ukrainian Academy of Medical Science", Kharkiv 61039, Ukraine.
}

\begin{abstract}
Correspondence to: Dr. Anna Shalimova, Department of Chronic Non-communicable Disease Prevention, Government Institution "National Institute of Therapy named by L.T. Malaya of National Ukrainian Academy of Medical Science", Kharkiv, 61039, Ukraine.E-mail: anna.shalimova83@gmail.com
\end{abstract} How to cite this article: Nemtsova V, Bilovol O, Ilchenko I, Shalimova A. Age-associated features of oxidative stress as marker of
vascular aging in comorbid course of hypertension and type 2 diabetes mellitus. Vessel Plus 2018;2:27.

http://dx.doi.org/10.20517/2574-1209.2018.48

Received: 25 Jun 2018 First Decision: 27 Jul 2018 Revised: 8 Aug 2018 Accepted: 31 Aug 2018 Published: 28 Sep 2018

Science Editor: Igor A. Sobenin Copy Editor: Cui Yu Production Editor: Zhong-Yu Guo

\begin{abstract}
Aim: To evaluate activity of oxidative stress (OS) as marker of vascular aging in different age groups of patients with combined course of arterial hypertension (HT) and type 2 diabetes mellitus (T2DM).

Methods: 126 patients (average age $57.8 \pm 6.2$ years) with stage II HT and compensated T2DM were divided into 2 subgroups: $2 \mathrm{a}(n=59)$ - aged $45-60$ years; $2 \mathrm{~b}(n=97)$ - aged 61-75 years; 30 patients with isolated stage II HT (comparison group), 20 practically healthy individuals (control group). The activity of antioxidative [glutathione peroxidase, sulfhydryl groups (SH-groups)] and oxidative [malonic dialdehyde (MDA)], 8-hydroxy-2-deoxyguanosine (8-OH-dG) systems in blood serum, were studied.
\end{abstract}

Results: A significant increase in MDA levels $(P<0.05)$ and $\mathrm{SH}$-groups $(P<0.05)$ compared with healthy volunteers was observed. Patients in $2 b$ group had lower MDA values than in $2 \mathrm{a}(6.25 \pm 0.33 \mu \mathrm{mol} / \mathrm{L}, 7.07 \pm 0.44 \mu \mathrm{mol} / \mathrm{L}$, respectively, $P>0.05)$. In the $2 \mathrm{~b}$ group, in comparison with 2 a patients, a decrease in thiol status was observed $(P>0.05)$. The level of $8-\mathrm{OH}-\mathrm{dG}$ was increased in patients with $\mathrm{HT}$ and T2DM, but there was also an age-associated increase in the average $8-\mathrm{OH}-\mathrm{dG}$ in the $2 \mathrm{~b}$ group.

Conclusion: The age-associated changes in the OS in comorbid course of HT and T2DM did not have significant differences. Nevertheless, the presence of correlations between various indexes that are included in the concept of "vascular

\footnotetext{
(@) $(0$

(C) The Author(s) 2018. Open Access This article is licensed under a Creative Commons Attribution 4.0 International License (https://creativecommons.org/licenses/by/4.0/), which permits unrestricted use, sharing, adaptation, distribution and reproduction in any medium or format, for any purpose, even commercially, as long as you give appropriate credit to the original author(s) and the source, provide a link to the Creative Commons license, and indicate if changes were made.
}

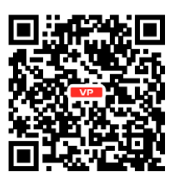


aging" and indicators of oxidant-antioxidant systems in different age groups allows us to make an assumption about the significant influence of the oxidative status on the status of vascular age, especially in the older age group persons.

Keywords: Hypertension, type 2 diabetes mellitus, oxidative stress, vascular aging

\section{INTRODUCTION}

A significant increase in the proportion of older people in the population of developed countries is accompanied by an increase in mortality from the main diseases of old age-diseases of the cardiovascular system, malignant neoplasms, neurodegenerative processes, reduced resistance to infection and diabetes mellitus.

According to the United Nations Organization prognosis, by 2025 the number of people over 60 will reach 1.2 billion ( $15 \%$ of the world's total population). Therefore, the concept of healthy aging, developed in 2001 by the United Nations Organization, is classified as one of the highest priority areas of medicine.

Aging is considered to be a natural physiological process. At the same time, there are data which demonstrate that physiological aging is observed in only $3 \%-6 \%$ of the human population while in other cases accelerated aging is observed. Aging is a biological process that develops with age and manifests as a gradual decrease in the adaptive capabilities of the organism. These changes can play a significant role in the development of different pathologies.

Medico-biological studies involving elderly, old people and centenarians are recognized as necessary to identify age-associated risk factors and specific markers that would optimize therapy for age-associated diseases, especially taking into consideration that cardio-vascular mortality remains high, despite therapeutic and prophylactic efforts. There is also a need to develop new pathophysiological models for a better understanding of cardiovascular risks (CVRs) based on new age-associated concepts.

Since age is a marker of the cumulative effect of risk factors and the overall integral index of the development of many chronic diseases [cardiovascular diseases (CVD), type 2 diabetes mellitus (T2DM), malignant diseases], Nilsson et al. ${ }^{[1]}$ proposed the concept of "early vascular aging", which is a new concept for studying patients with high CVR or patients with early family manifestations of cardiovascular events. "Vascular age" (VA) generally includes many determinants, the main ones of which are: endothelial dysfunction, pulse wave velocity (PWV), central arterial pressure and carotid intima-media complex thickness (CIMCT). These parameters can be considered as "tissue biomarkers" of vascular lesions, which may be more sensitive than "circulating biomarkers" (e.g., C-reactive protein, hyperglycemia, dyslipidemia) and in combination with classical risk factors show better additional results predicting cardiovascular

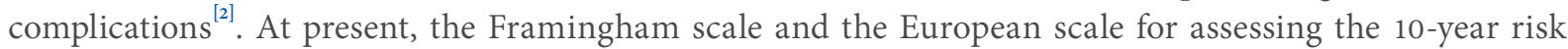
of cardiovascular mortality (SCORE) are widely used, in which age is one of the most important CVR determinants and is associated with a number of morphological and functional changes in vessels ${ }^{[3]}$.

As knowledge accumulates, it is becoming increasingly evident that aging and major chronic age-related diseases have the same basic molecular and cellular mechanisms ${ }^{[4]}$. It is believed that in the first place they are associated with mild chronic systemic inflammation. To denote this phenomenon a group of scientists led by Franceschi et al ${ }^{[5]}$ proposed the term "inflammaging". Today inflammaging is a widely accepted theory of aging. Global age-related systemic inflammation in many organs is involved in the pathogenesis of the most associated diseases, but until now it has not been fully determined whether these conditions are the cause or consequence of age-related systemic inflammation. Inflammation is one of the central pathogenetic mechanisms at all stages of development of atherosclerosis and its complications. What 
mechanisms are involved in the development of age-associated chronic inflammation is not definitively established.

The age-associated increase in oxidative stress can contribute to the development of chronic inflammation and the progression of various diseases. Today the theory of oxidative stress is considered one of the most popular theories explaining not only aging, but also the initiation, as well as the progression of many modern diseases, in particular cardiovascular and diabetes mellitus. Recently, oxidative stress has been actively studied in order to better understand the mechanisms of protection and the relationship between oxidative damage and the aging process ${ }^{[6]}$.

Population aging became the leading demographic feature of Ukraine as well ${ }^{[7]}$. Hypertension (HT), $\mathrm{T} 2 \mathrm{DM}$, ischemic heart disease (IHD), cerebral vascular disease with different severity of clinical symptoms and in different combinations are the most common in elderly people ${ }^{[4,7]}$.

The purpose of this study was to evaluate plasma parameters of oxidant-antioxidant systems as markers of vascular aging in patients of different age groups with a combined course of arterial HT and T2DM.

\section{METHODS}

126 patients (55 males and 71 females) from 45-75 years old (mean age: $57.8 \pm 6.2$ years) with stage II HT (mean duration of disease 10.2 \pm 3.7 years) and well controlled T2DM (mean duration of disease: $4.1 \pm 2.4$ years) were examined and according to the current World Health Organization (WHO) age classification ${ }^{[8]}$ were divided into 2 subgroups: group $2 \mathrm{a}(n=59)$ - patients with HT in combination with T2DM aged 4560 years; group $2 \mathrm{~b}(n=97)$ - patients with a combined course of HT and T2DM aged 61-75 years. As a comparison group, patients with isolated stage II H $(n=30)$, identical in age and sex to the main group (average age $53.8 \pm 4.6$ years) were studied. The control group consisted of 20 healthy individuals, matched for age and sex.

The study did not include patients with symptomatic HT, uncontrolled HT, type 1 diabetes, decompensated $\mathrm{T} 2 \mathrm{DM}$ and other endocrine disorders, clinical signs of IHDs or severe concomitant chronic diseases. The excluding criteria were also: taking iodine medications, glucocorticoids, amiodarone, lithium medications, and medications containing estrogens and pregnancy. For patient selection, the diagnostic criteria of HT approved by the European recommendations on diagnosis and treatment of $\mathrm{HT}^{[9]}$ were used. The diagnosis of T2DM was established according to the approved by order of the Ministry of Health of Ukraine dated on 21.12.2012 № 1118 "On Approval and implementation of medical-technological documents for the standardization of medical aid in type 2 diabetes" ${ }^{\text {"[10] }}$ and in accordance with the recommendations of the American Diabetes Association and the European Association for the study of diabetes ${ }^{[11]}$.

On a background of dietary recommendations, all patients received basic therapy in accordance with international and national recommendations for the management of patients with appropriate pathology ${ }^{[9-11]}$. Before being included in the study, all patients had been receiving antihypertensive therapy for at least 6 months in individually selected doses with the use of angiotensin - converting enzyme (ACE) inhibitors or angiotensin II receptors blockers (ACE inhibitors/ARBS), diuretics (indapamide or torasemide). Some of the patients received calcium antagonists (amlodipine or lercanidipine). As an antidiabetic therapy, patients with T2DM received metformin in individually selected doses from 1000$2000 \mathrm{mg}$ per day, 49 patients (29.51\%) additionally were using sulfonylurea derivatives.

Blood pressure (BP) levels were assessed in all patients by means of blood pressure obtained from three measurements at 2-min intervals in a sitting position. 
Determination of total cholesterol (TC), triglycerides (TG) and cholesterol of high density lipoproteins (HDL cholesterol) were performed in serum enzymatically by photocolorimetric method with sets produced by Human (Germany). The content of cholesterol in the low density lipoprotein (LDL cholesterol) was calculated by the formula of Friedewald W. T. with consideration of measurement in mmol/L: LDL cholesterol $=$ cholesterol $-($ HDL cholesterol $+\mathrm{TG} / 2.22)$.

Determination of the concentration of fasting glucose was performed by the glucose oxidase method using analyzer Humolizer (made in Germany). The level of glycated hemoglobin (HbA1c) was measured by enzyme immunoassay (ELISA) using a set of reagents Hummer (USA). To determine the insulin resistance (IR) index HOMA-IR was used, which was calculated with the formula: [(Glucose fasting) $\times$ (fasting insulin)] $\mathrm{mmoL} / \mathrm{mL} / 22.5$.

Besides the indicators of carbohydrate and lipid metabolism all patients underwent measurement of the concentration of insulin in blood serum by the method of ELISA using a kits DRG Instrument Gmbh (Germany) on a semi-automatic ELISA analyzer "ImmunoChem-2100", HighTechnology, Inc. (USA).

To study the antioxidative system, the activity of glutathione peroxidase (GPO) and the level of sulfhydryl groups (SH-groups) were assessed. GPO plays an important role in protecting biological cell membranes against oxidative damage by increasing the concentration of reduced glutathione (oxidised glutathione ratio - GSSG-R) in the process of aerobic glycolysis. SH-groups are the organic compounds that contain a sulphydryl group. Among all the antioxidants that are available in the body, they constitute the major portion of the total body antioxidants and they play a significant role in defense against reactive oxygen species (ROS). The level of malonic dialdehyde (MDA) was used as a marker of the lipid peroxidation and oxidative system activity. The activity of GPO (KF 1.11.1.9) in Ethylenediaminetetraacetic acid (EDTA)-hemolysate was determined by the decrease in the content of reduced glutathione during a 5-min incubation of a test sample of hemolysate in the presence of oxidizing substrate - cumene hydroperoxide by the photometric method ${ }^{[12]}$. The SH-groups and MDA were determined in serum using a photometric method ${ }^{[12]}$. The following reagents were used: thiobarbituric acid (Organika, Germany), dithiobisnitrobenzoic acid (Merck, Germany), restored glutathione (Sigma-Aldrich, Germany), cumene hydroperoxide (Merck, Germany). The determination of 8-hydroxy-2-deoxyguanosine (8-OH-dG) in blood serum, as one of the biomarkers of oxidative damage, was carried out by ELISA with kits "Bio-Vendor" (Czech Republic).

Ultrasound examination of the common CIMCT was performed according to the standard procedure on the device "LOGIQ5".

The results obtained are presented as the average value of parameters $(M)$ and standard error (m). Processing of statistical data was performed using the software package "Statistics for Windows 8.0". The student criterion and Pearson's chi-squared test were used to estimate the differences between groups with normal distribution. The differences were considered statistically significant at $P<0.05$.

The study was performed in compliance with the basic provisions of the Helsinki declaration of the world medical association on ethical principles of scientific and medical research involving humans (1964-2000) and the order of the ministry of health of Ukraine dated 23.09.2009 № 690. The article is a fragment of the research work of the department of clinical pharmacology and internal medicine of Kharkiv National Medical University "Optimization of diagnosis and treatment of comorbid pathology (HT and diabetes mellitus type 2) on the basis of evaluation of cardio-hemodynamics, metabolism and pharmacogenetic 


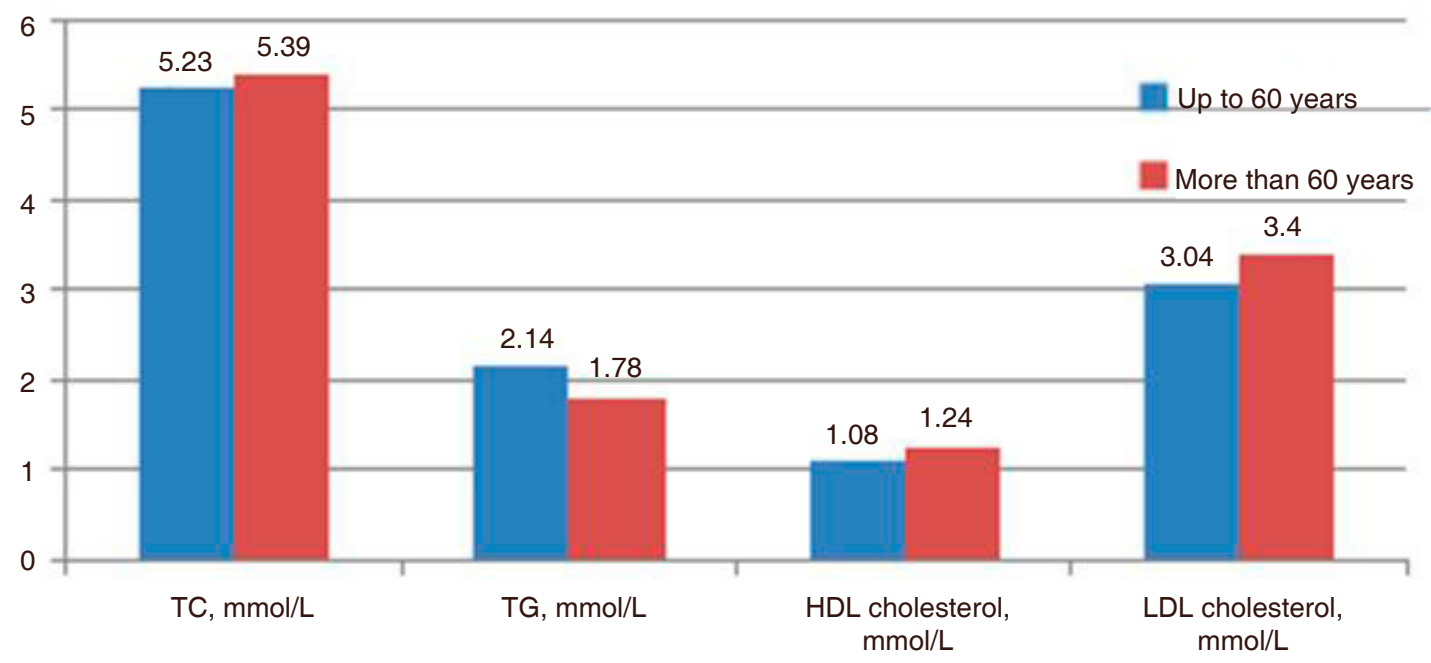

Figure 1. The state of lipid metabolism in patients with $\mathrm{AH}$ and DM2T, depending on the age group. TC: total cholesterol; TG: triglycerides; HDL cholesterol: cholesterol of high density lipoproteins; LDL cholesterol: cholesterol of low density lipoproteins

analysis". The study was approved by the commission on bioethics at the Kharkiv National Medical University, consistent with the principles outlined in the Helsinki declaration.

\section{RESULTS}

Comparative analysis of lipid and carbohydrate metabolism parameters, as was expected, showed the presence of dyslipidemia both among patients with isolated HT and in the combined course of HT and $\mathrm{T} 2 \mathrm{DM}$ in comparison with controls. Inclusion of compensated T2DM to HT was not accompanied by a significant aggravation of lipid metabolism disorders, but increased the index of IR almost 2 -fold compared with patients with isolated HT and 4-fold compared with the control group (group 1: $4.40 \pm 0.51$, group 2: $8.26 \pm 0.68$, control: $2.23 \pm 0.36$ respectively, $P<0.05)$.

The distribution of patients with HT and T2DM in the 2 age categories did not reveal significant differences in the disorders of both lipids, except HDL cholesterol, and carbohydrate metabolism [Figure 1]. In the $2 \mathrm{~b}$ subgroup patients, despite a higher level of fasting glucose (2a subgroup: $8.59 \pm 0.72 \mathrm{mmoL} / \mathrm{L}$ and $2 \mathrm{~b}: 9.15 \pm$ $0.67 \mathrm{mmoL} / \mathrm{L}$, respectively, $P>0.05$ ), there was a decrease in the signs of insulin resistance (HOMA-IR: 2a: $8.88 \pm 1.15$ and 2 b: $7.92 \pm 0.86$, respectively, $\mathrm{P}>0.05$ ). It is believed that the increase in IR leads to the depletion of antioxidant protection. In our work in patients with comorbid pathology with an increase in the age group, there was a unidirectional decrease in the signs of both IR and indexes of both oxidative and antioxidant systems [Table 1]. In patients of the older age group, the correlation analysis revealed the presence of an average positive relationship between GPO and HOMA-IR $(P=0.046, r=0.374)$.

When evaluating the carotid intima-media thickness (CIMT) we were guided by the data obtained in the Atherosclerosis Risk in Communities (ARIC) study, in which it was demonstrated that the calculation of VA, taking into account gender, age and race, can be made on the basis of the CIMT measurement ${ }^{[2]}$. Stein et al. ${ }^{[13]}$ have shown that the assessment of VA using a non-invasive CIMT measurement, can more accurately determine the age as one of the main indicators in the evaluation individual risk of cardiovascular disease.

As is known, the presence of $\mathrm{HT}$ and $\mathrm{T} 2 \mathrm{DM}$ themselves are characterized by a tendency to increase the CIMT, which we observed in our patients, although it is worth noting that this increase was in comparison 
Table 1. Age-associated comparative assessment of oxidative-antioxidant systems in patients with H and DM2T

\begin{tabular}{lcc}
\hline Index & 2a subgroup $(\boldsymbol{n}=\mathbf{5 9})$ & 2b subgroup $(\boldsymbol{n}=\mathbf{9 7})$ \\
\hline $8-\mathrm{OH}-\mathrm{dG}, \mathrm{ng} / \mathrm{L}$ & $14.07 \pm 1.03$ & $17.23 \pm 0.97^{\star}$ \\
$\mathrm{GPO}, \mu \mathrm{mol} / \mathrm{min} / \mathrm{gHb}$ & $5.73 \pm 0.36$ & $5.23 \pm 0.23$ \\
$\mathrm{MDA}, \mu \mathrm{mol} / \mathrm{L}$ & $7.07 \pm 0.44$ & $6.25 \pm 0.33$ \\
$\mathrm{SH}-\mathrm{groups}, \mu \mathrm{mol} / \mathrm{L}$ & $584.38 \pm 14.56$ & $567.36 \pm 15.03$ \\
$\mathrm{CIMT} \mathrm{RCCA}, \mathrm{mm}$ & $0.08 \pm 0.00$ & $0.08 \pm 0.00$ \\
CIMT LCCA, mm & $0.08 \pm 0.00$ & $0.08 \pm 0.00$ \\
Insulin, $\mu \mathrm{IU} / \mathrm{mL}$ & $24.55 \pm 4.24$ & $19.71 \pm 2.17$ \\
$\mathrm{HOMA}-\mathrm{IR}$ & $8.88 \pm 1.15$ & $7.92 \pm 0.86$ \\
$\mathrm{SBP}, \mathrm{mmHg}$ & $152.92 \pm 5.85$ & $143.64 \pm 2.82$ \\
DBP, mmHg & $92.50 \pm 3.29$ & $88.88 \pm 1.75$ \\
PBP, mmHg & $60.42 \pm 3.72$ & $54.76 \pm 2.15$ \\
\hline
\end{tabular}

${ }^{\star} P<0.05$ : compared to the 2a group; HOMA-IR: insulin resistance index; MDA: malonic dialdehyde; GPO: glutathione peroxidase; SHgroups: sulfhydryl groups; 8-OH-dG: 8-hydroxy-2-deoxyguanosine; CIMT RCCA: carotid intima-media thickness of right common carotid artery; CIMT LCCA: carotid intima-media thickness of left common carotid artery; SBP: systolic blood pressure; DBP: diastolic blood pressure; PBP: pulse blood pressure

with control group, and in 67\% not reach the threshold for the European recommendations (CIMT thickness $\leq$ $0.9 \mathrm{~mm})^{[14]}$. The subdivision of patients with HT and T2DM into age groups was accompanied by a slight increase in this parameter, which did not reach significance (2a subgroup - CIMT of right common carotid artery (RCCA) $-0.086 \pm 0.004 \mathrm{~mm}$, CIMT of left common carotid artery (LCCA) $-0.086 \pm 0.003 \mathrm{~mm}, 2 \mathrm{~b}$ subgroup - CIMT RCCA - $0.088 \pm 0.003 \mathrm{~mm}$ and CIMT LCCA - $0.087 \pm 0.002 \mathrm{~mm}$, respectively, $P>0.05)$. Ultrasound examination of carotid arteries revealed the presence of atherosclerotic plaques in 39 patients (30.95\%), 5 of which (12.82\%) belong to the 2 a subgroup, and 34 patients (87.18\%) - 2b subgroup.

Oxidative stress is defined as an imbalance between the concentration of oxidation products and the activity of antioxidant processes in the body. Oxidative stress promotes the oxidation of a number of molecules, such as DNA, lipids, and proteins, which are associated with various processes, including aging.

Despite the available data on the beneficial effect of modern antihypertensive ${ }^{[15]}$ and antidiabetic ${ }^{[16]}$ therapy on oxidative stress, the results of our study demonstrate the presence of intensive OS in patients with the combined course of HT and T2DM. This manifests in a significant increase in MDA levels $(P<0.05)$, and a decrease in levels of SH-groups $(P<0.05)$ compared with healthy volunteers.

MDA is the main end product in the process of lipid peroxidation. Data from recent years suggest using the MDA level as a marker of the risk of complications in patients with T2DM, especially inadequately compensated $^{[17]}$.

In the studies of Carracedo et al ${ }^{[18]}$ it was shown that people with age showed a significant increase in MDA. In our work, patients in the older age group had lower MDA values than those under $60(6.25 \pm 0.33 \mu \mathrm{moL} / \mathrm{L}$ and $7.07 \pm 0.44 \mu \mathrm{moL} / \mathrm{L}$, respectively, $P>0.05)$.

The main role in protecting against the influence of OS is played by an antioxidant system (AOS), one of the main components of which is the thiol disulfide system. An important biomarker reflecting the state of this system is the organism thiol status. Thiol status indicates the total level of SH-groups of proteins and free SHgroups. In patients with the combined course of HT and T2DM [Table 2], there was a significant decrease in thiol status in comparison with the control group $(573.52 \pm 10.91 \mu \mathrm{moL} / \mathrm{L}$ and $712.26 \pm 11.08 \mathrm{mmoL} / \mathrm{L}$, respectively, $P<0.05$ ). 
Table 2. Indexes of lipid, carbohydrate metabolism, oxidant and antioxidant systems in patients with isolated hypertension, combination of $\mathrm{H}$ and DM2T compared with controls

\begin{tabular}{|c|c|c|c|}
\hline Index & Control $(n=20)$ & Isolated H (group1, $n=30$ ) & H with DM2T (group2, $n=126$ ) \\
\hline TC, mmol/L & $4.77 \pm 0.52$ & $5.95 \pm 0.23$ & $5.34 \pm 0.21$ \\
\hline $\mathrm{TG}, \mathrm{mmol} / \mathrm{L}$ & $1.03 \pm 0.30$ & $1.63 \pm 0.13$ & $1.91 \pm 0.14$ \\
\hline VLDL cholesterol, $\mathrm{mmol} / \mathrm{L}$ & $0.54 \pm 0.22$ & $0.72 \pm 0.06$ & $0.85 \pm 0.08$ \\
\hline HDL cholesterol, mmol/L & $1.45 \pm 0.30$ & $1.41 \pm 0.06$ & $1.18 \pm 0.04^{\star}$ \\
\hline LDL cholesterol, mmol/L & $2.6 \pm 0.33$ & $3.7 \pm 0.24$ & $3.28 \pm 0.21$ \\
\hline Glucouse, $\mathrm{mmol} / \mathrm{L}$ & $4.62 \pm 1.08$ & $5.45 \pm 0.12$ & $8.25 \pm 0.30^{\star}$ \\
\hline $\mathrm{HbA} 1 \mathrm{c}, \%$ & $4.62 \pm 1.08$ & $6.22 \pm 0.15$ & $7.32 \pm 0.20^{\star}$ \\
\hline Insulin, $\mu \mathrm{IU} / \mathrm{mL}$ & $9.84 \pm 2.20$ & $19.77 \pm 2.06$ & $22.89 \pm 2.20$ \\
\hline HOMA-IR & $2.23 \pm 0.36$ & $4.40 \pm 0.51$ & $8.26 \pm 0.68^{\star}$ \\
\hline $\mathrm{MDA}, \mu \mathrm{mol} / \mathrm{L}$ & $4.07 \pm 0.22$ & $6.11 \pm 0.31$ & $6.55 \pm 0.27$ \\
\hline $\mathrm{GPO}, \mu \mathrm{mol} / \mathrm{min} / \mathrm{gHb}$ & $406.20 \pm 31.2$ & $346.80 \pm 15.01$ & $324.60 \pm 12.01$ \\
\hline $\mathrm{SH}$-groups, $\mu \mathrm{mol} / \mathrm{L}$ & $712.26 \pm 11.08$ & $570.54 \pm 12.64$ & $573.52 \pm 10.91$ \\
\hline $8-\mathrm{OH}-\mathrm{dG}, \mathrm{ng} / \mathrm{L}$ & $6.66 \pm 0.97$ & $16.26 \pm 0.83$ & $15.89 \pm 0.76$ \\
\hline
\end{tabular}

$\star P<0.05$ : compared to the 1st group; TC: total cholesterol; TG: triglycerides; VLDL cholesterol, HDL cholesterol: cholesterol of high density lipoproteins; LDL cholesterol: cholesterol of low density lipoproteins; HbA1c: glycated hemoglobin; HOMA-IR: insulin resistance index; MDA: malonic dialdehyde; GPO: glutathione peroxidase; SH-groups: sulfhydryl groups; 8-OH-dG: 8-hydroxy-2-deoxyguanosine

This trend persisted even when patients were divided into age groups: in patients of the older age group in comparison with younger patients a decrease in thiol status was observed $(P>0.05)$. These changes were observed against the background of an insignificant age-dependent decrease of GPO, which indicates a decrease of the antioxidant protective force [Table 1].

Previously, it was shown that guanine is the most oxidizable of the four bases included in the DNA structure $^{[19]}$. Its oxidation product, as a result of ROS exposure, is $8-\mathrm{OH}-\mathrm{dG}$, which can be determined in various biological tissues and liquids. In our study the correlation analysis revealed 8-OH-dG bonds practically only in the group of individuals under 60 years: the average positive correlation between 8 - $\mathrm{OH}$ $\mathrm{dG}$ and CIMT $(P=0.038, r=-0.569)$, a high positive relationship between $8-\mathrm{OH}-\mathrm{dG}$ and SBP $(P=0.027, r=$ $0.765) 8-\mathrm{OH}-\mathrm{dG}$ and PBP $(P=0.046, r=0.715)$.

The correlation analysis also revealed the age-dependence of various correlations between the studied parameters. Thus, in the group under 60 years a weak negative relationship was found between the SHgroups and the TG levels $(P=0.002, r=-0.04)$ and HDL cholesterol levels $(P=0.042, r=-0.206)$. In the combined course of HT and T2DM in patients of the older age group the correlation analysis revealed the presence of an average positive association between 8-OH-dG levels and HDL cholesterol $(P=0.045$, $r=0.543)$, GPO and TC $(P=0.026, r=0.405)$, GPO and LDL cholesterol $(P=0.027, r=0.410)$, GPO and HOMA-IR $(P=0.046, r=0.374)$.

\section{DISCUSSION}

It is known that oxidative stress increases with age and its progressive development can be considered as one of the aging markers. It is generally accepted that an increase in OS during aging is a consequence of a decrease in the effectiveness of antioxidant protection. The quantitative determination of 8-OHdG is suggested as one of the markers of free-radical processes occurring in the body under normal circumstances and with the development of various pathological processes. It is believed that an increased level of $8-\mathrm{OH}-\mathrm{dG}$ is associated with the aging process, as well as with many pathological conditions, including diabetes mellitus and HT. In the studies of Wua et al. ${ }^{[19]}$ the correlations between the level of oxidative DNA damage and the severity of diabetic nephropathy and retinopathy were demonstrated. 
In our study, we observed not only an increase in this index in patients with HT and T2DM compared to the control group, but there was also an age-associated increase in the average $8-\mathrm{OH}-\mathrm{dG}$ in the $2 \mathrm{~b}$ age group, which coincides with the results of Wang et al ${ }^{[20]}$, who demonstrated a significant relationship between the plasma level of 8-OHdG and the age of the studied subjects.

Our results indicate that the combined course of $\mathrm{HT}$ and $\mathrm{T} 2 \mathrm{DM}$ is characterized by the presence of pronounced oxidative stress, which manifests in a significant increase in the intensity of lipid peroxidation, the marker of DNA damage which increased against the background of a decreased activity of antioxidant protection.

Despite the fact that the age-associated changes in oxidative stress in comorbid course of HT and T2DM did not have significant differences, the presence of correlations between various indexes that are included in the concept of "vascular aging" and indicators of oxidant-antioxidant systems in different age groups allows us to make an assumption about the significant influence of the oxidative status on the status of VA, especially in the older age group persons.

Given that elderly age is generally characterized by polymorbidity and with age the risk of age-associated diseases development, including HT and T2DM and their complications, increases, one active area of research is to study the peculiarities of oxidative stress development in older age groups.

Thus, to date the concept of VA allows us to take a new view at the assessment of CVR: on the one hand, as a biological model of aging, on the other, it makes it possible to analyze the state of many risk factors in different age groups.

\section{DECLARATIONS}

\section{Authors' contributions}

Study design, manuscript review: Nemtsova V, Bilovol O

Development of methodology: Nemtsova $\mathrm{V}$

Collection of data, analysis and/or interpretation of data, writing (not revising) all or sections of the manuscript: Nemtsova V, Bilovol O, Ilchenko I, Shalimova A

Supervision: Bilovol O

\section{Availability of data and materials}

Not applicable.

\section{Financial support and sponsorship}

None.

\section{Conflicts of interest}

All authors declared that there were no conflicts of interest.

\section{Ethical approval and consent to participate}

The study protocol was supported by the Ethics Committee of the Kharkiv National Medical University, Ukraine.

\section{Consent for publication}

Each patient was informed the study and gave their consent.

\section{Copyright}

(c) The Author(s) 2018. 


\section{REFERENCES}

1. Nilsson PM, Boutouyrie P, Laurent S. Vascular aging: a tale of EVA and ADAM in cardiovascular risk assessment and prevention. Hypertension 2009;54:3-10.

2. Sinkevich DA, Protasov KV, Dzyzinsky AA. The concept of vascular age as a new approach to evaluation of cardiovascular risk. Siberian Med J 2011;6:9-13.(in Russian)

3. Lakatta EG, Levy D. Arterial and cardiac aging: major shareholders in cardiovascular disease enterprises: part I: aging arteries: a "set up" for vascular disease. Circulation 2003;107:139-46.

4. Luchikhina LV, Mendel OI, Mendel V, Golukhov GN. Osteoarthritis and age. Role of aging in the etiology and pathogenesis of the disease. Mod rheumatology 2017;11:4-11. (in Russian)

5. Franceschi C, Capri M, Monti D, Giunta S, Olivieri F, Sevini F, Panourgia MP, Invidia L, Celani L, Scurti M, Cevenini E, Castellani GC, Salvioli S. Inflammaging and anti-inflammaging: a systemic perspective on aging and longevity emerged from studies in humans. Mech Ageing Dev 2007;128:92-105.

6. Hammad EV, Belousova ON, Khmelnitsky AV, Poltoratsky AN, Shchekaturov AA. Modern biomaterials of aging for stratification of risks associated with development of age-associated diseases (review) // Scientific Bulletins of BelSU. Series: medicine. Available from: https://cyberleninka.ru/article/n/sovremennye-biomaterialy-stareniya-dlya-stratifikatsii-riskov-razvitiya-vozrast-assotsiirovannyhzabolevaniy-obzor-literatury. [Last accessed on 19 Sep 2018]

7. Kovalenko VM, Kornatsky VM. Diseases of the circulatory system as a medical and social and socio-political problem (analytical and statistical manual). Kiev; 2014. p. 279. (in Ukrainian)

8. Age classification of the World Health Organization. Available from: https://citifox.ru/2016/05/05/vozrastnaya-klassifikaciyavsemirnoy/. [Last accessed on 19 Sep 2018]

9. Mancia G, Fagard R, Narkiewicz K, Redón J, Zanchetti A, Böhm M, Christiaens T, Cifkova R, De Backer G, Dominiczak A, Galderisi M, Grobbee DE, Jaarsma T, Kirchhof P, Kjeldsen SE, Laurent S, Manolis AJ, Nilsson PM, Ruilope LM, Schmieder RE, Sirnes PA, Sleight P, Viigimaa M, Waeber B, Zannad F; Task Force Members. 2013 ESH/ESC guidelines for the management of arterial hypertension: the task force for the management of arterial hypertension of the European Society of Hypertension (ESH) and of the European Society of Cardiology (ESC). J Hypertens 2013;31:1281-357.

10. Ministry of the Health of Ukraine. Order of the Ministry of Health of Ukraine dated 21.12.2012 № 1118 “on approval and implementation of medical-technological documents for the standardization of medical aid in type 2 diabetes". Available from: https:// medprosvita.com.ua/nakaz-moz-ukrayini-vid-21-12-2012-n-1118-pro-zatver/. [Last accessed on 19 Sep 2018]

11. Inzucchi SE, Bergenstal RM, Buse JB, Diamant M, Ferrannini E, Nauck M, Peters AL, Tsapas A, Wender R, Matthews DR. Management of hyperglycemia in type 2 diabetes, 2015: a patient-centered approach: update to a position statement of the American Diabetes Association and the European Association for the study of diabetes. Diabetes Care 2015;38:140-9.

12. Arutyunov AV, Dubinina EE, Zybina N.N. Methods for assessing free radical oxidation and the body's antioxidant system. Guidelines. St. Petersburg: IKF "Foliant"; 2000. p. 104 (in Russian)

13. Stein JH, Fraizer MC, Aeschlimann SE, Nelson-Worel J, McBride PE, Douglas PS. Vascular age: integrating carotid intima-media thickness measurements with global coronary risk assessment. Clin Cardiol 2004;27:388-92.

14. Ostroumova OD, Zhukova OV, Erofeeva AG, Tolkalenov AV. Thickness of carotid arteries intima-media complex in patients with $\mathrm{H}$ the possibility of a fixed combination Logimax. Rus Med J 2009;8:548 (in Russian)

15. E.A.Prochorovich. Hypotensive therapy: a new combination and new possibilities. Consilium Medicum 2013;10:121-5.

16. Alireza Esteghamati, Delaram Eskandari, Hossein Mirmiranpour, Sina Noshad, Mostafa Mousavizadeh, Mehdi Hedayati, Manouchehr Nakhjavan. Randomized clinical trial: the effect of metformin on markers of oxidative stress and antioxidant reserves in patients with newly diagnosed type 2 diabetes. Obes Metabolism 2012;3:41-2.

17. Lodovici M, Bigagli E, Luceri C, Mannucci E, Rotella CM, Raimondi L. Gender-related drug effect on several markers of oxidation stress in diabetes patients with and without complications. Eur J Pharmacol 2015;766:86-90.

18. Carracedo J, Ramírez-Carracedo R, Martínez de Toda I, Vida C, Alique M, De la Fuente M, Ramírez-Chamond R. Protein carbamylation: a marker reflecting increased age-related cell oxidation. Int J Mol Sci 2018; doi: 10.3390/ijms19051495.

19. Wu LL, Chiou CC, Chang PY, Wu JT. Urinary 8-OH-dG: a marker of oxidative stress to DNA and a risk factor for cancer, atherosclerosis and diabetics. Clin Chim Acta 2004;339:1-9.

20. Wang CC, Chen WL, Liou SH. P081 The difference between plasma and urinary 8-hydroxy-2-deoxyguanosine biomarkers measured by liquid chromatrography tandem mass spectrometry. Occup Environ Med 2016;73:A147. 